12

\title{
Моделирование динамики резистивной шланговой неустойчивости релятивистского электронного пучка, распространяющегося в омическом плазменном канале произвольной проводимости
}

\author{
() Е.К. Колесников, А.С. Мануйлов, В.С. Петров
}

Санкт-Петербургский государственный университет, 199034 Санкт-Петербург, Россия

e-mail: a.manuylov@spbu.ru, man06@mail.ru

(Поступило в Редакцию 25 янваля 2017 г. В окончательной редакции 20 марта 2017 г.)

Исследована пространственная динамика резистивной шланговой неустойчивости релятивистского электронного пучка в случаях, когда время зарядовой нейтрализации много больше, порядка и много меньше времени токовой компенсации. Получено, что наибольшим пространственным инкрементом нарастания указанная неустойчивость обладает в случае, когда время зарядовой нейтрализации порядка скинового времени.

DOI: 10.21883/JTF.2017.11.45136.2180

\section{Введение}

В последние несколько десятилетий внимание исследователей привлекают вопросы распространения релятивистских электронных пучков (РЭП) в плотных и разреженных газоплазменных средах. Особое место в этой проблеме занимает исследование резистивных крупномасштабных неустойчивостей, являющихся одним из деструктивных факторов при транспортировке РЭП в указанных средах. В частности, в работах [1-11] исследовалось развитие наиболее опасной для распространения пучка резистивной шланговой неустойчивости (РШН), которая представляет собой нарастающие по амплитуде изгибные колебания РЭП, которые могут достаточно быстро привести к развалу пучка.

В большей части исследований по динамике РШН РЭП [5-8] предполагается, что скалярная омическая проводимость фоновой газоплазменной среды весьма велика. При этом выполняется условие

$$
\frac{4 \pi \sigma R_{b}}{c} \gg 1,
$$

где $\sigma$ - характерная проводимость плазменного канала в газоплазменной среде, $R_{b}-$ характерный радиус пучка, $c$ - скорость света.

Однако возможны ситуации, когда условие (1) не выполнено. В частности, данное условие нарушено в головной части пучка, когда он распространяется в нейтральном газе и наработка проводимости пучком в результате ударной ионизации не успевает создать достаточно высокую омическую проводимость. В связи с этим в настоящей работе рассмотрен случай произвольной проводимости омического плазменного канала. При этом построена модель динамики РШН РЭП, когда выполнено условие $\tau_{m} \sim \tau_{c}$ и $\tau_{m} \ll \tau_{c}$, где

$$
\tau_{m} \approx \frac{4 \pi \sigma R_{b}^{2}}{c^{2}}, \quad \tau_{c} \approx \frac{1}{4 \pi \sigma}
$$

- соответственно монопольное скиновое время и характерное время зарядовой компенсации.

Рассмотрим транспортировку РЭП по омическому плазменному каналу вида

$$
\sigma(r, \xi)= \begin{cases}\sigma_{0}(\xi), & r \leq R_{c h} \\ 0, & r>R_{c h}\end{cases}
$$

где $\xi=\beta c \tau, \quad \tau=t-z /(\beta c)-$ сдвинутое время, $\beta=v_{z} / c, v_{z}-$ продольная компонента скорости частиц пучка (РЭП предполагается моноскоростным в продольном направлении), $t-$ время, $R_{c h}-$ радиус плазменного канала.

Далее для простоты будем полагать, что $\sigma_{0}$ не зависит от $\xi$. Кроме того, будем считать, что плотность тока пучка в равновесии может быть представлена как

$$
J_{b z 0}(r, \xi)= \begin{cases}F(\xi) J_{0}\left(S_{0} r / R_{c h}\right), & r \leq R_{c h}, \\ 0, & r>R_{c h},\end{cases}
$$

где $J_{0}-$ функция Бесселя нулевого порядка, $S_{0}-$ первый нуль указанной функции Бесселя, $F(\xi)=$ $=I_{b 0} P(\xi) /\left(\pi R_{c h}^{2}\right) ; \quad I_{b 0}, P(\xi)$ - полный ток пучка и функция его модуляции.

С помощью невозмущенных и возмущенных компонент редуцированных уравнений Максвелла, полученных в $[7,8]$, нетрудно сформулировать следующие уравнения:

$$
\begin{gathered}
\xi_{m} \xi_{c} \frac{\partial^{2} \Psi_{f}}{\partial \xi^{2}}+\left(\xi_{m}+\xi_{c}\right) \frac{\partial \Psi_{f}}{\partial \xi}+\Psi_{f}=\Psi_{b} \\
\xi_{m} \xi_{c} \frac{\partial^{2}}{\partial \xi^{2}}\left(\Psi_{f} D\right)+\left(\xi_{m}+\xi_{c}\right) \frac{\partial}{\partial \xi}\left(\Psi_{f} D\right)+\Psi_{f} D=\Psi_{b}(\xi) Y
\end{gathered}
$$

где

$$
\begin{gathered}
\Psi_{f}(\xi)=\frac{A_{0}^{*}(r, \xi)}{J_{0}\left(S_{0} r / R_{c h}\right)}, \quad A_{1}^{*}=-D \frac{\partial A_{0}^{*}}{\partial r} \\
\Psi_{b}=\frac{4 I_{b 0}}{c S_{0}^{2}} P(\xi), \quad \xi_{m}=\frac{4 \pi R_{c h}^{2}}{S_{0}^{2} c} \sigma_{0}, \quad \xi_{c}=\frac{c}{4 \pi \sigma_{0}} .
\end{gathered}
$$



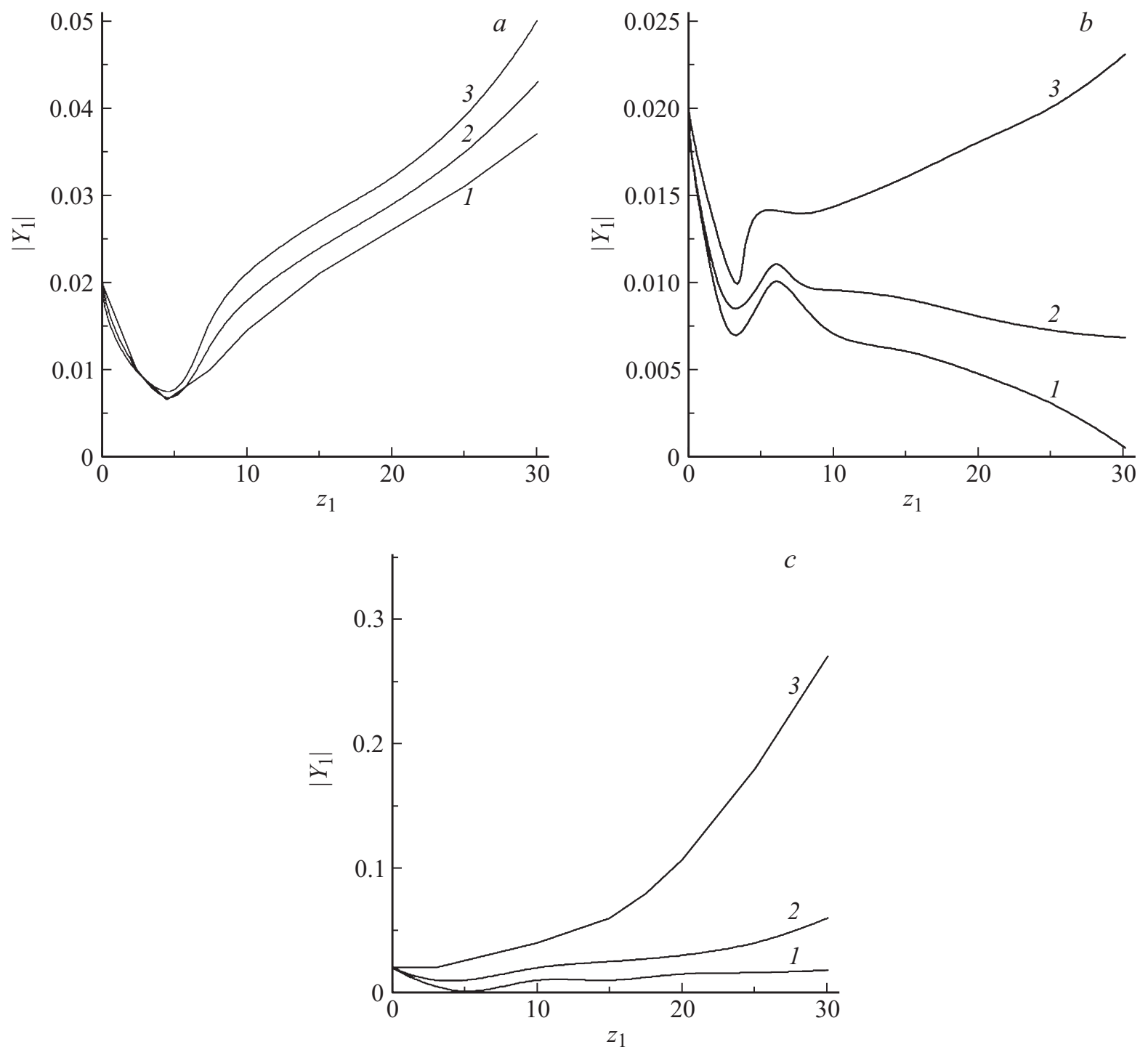

Зависимости $\left|Y_{1}\right|$ от безразмерной координаты $z_{1}$ при значениях частоты $\omega_{1}: a-0.2, b-1.2, c-1.6$. На всех рисунках кривая 1 соответствует $\eta=\xi_{m} / \xi_{c}=30,2-0.2,3-1$.

Здесь $A^{*}=A_{z}-\varphi\left(A_{z}-z\right.$-компонента векторного потенциала коллективного электромагнитного поля системы плазма-пучок, $\varphi$ - скалярный потенциал коллективного электрического поля), индексы „0“ и „1“ относятся соответственно к невозмущенным и возмущенным величинам, $\xi_{m}$ и $\xi_{c}-$ соответственно монопольная скиновая длина (длина, на которой происходит магнитная нейтрализация) и пространственный масштаб зарядовой компенсации в рассматриваемой задаче.

Используя модель жесткого пучка на линейной стадии развития РШН, в данном случае имеем

$$
\frac{\partial^{2} Y}{\partial z^{2}}=\frac{\Psi_{f}(\xi)}{\Psi_{b}(\xi)} k_{s}^{2}(D-Y)-\alpha_{p h} \sqrt{\left|\frac{\Psi_{f}(\xi)}{\Psi_{b}(\xi)}\right|} k_{s} \frac{\partial Y}{\partial z},
$$

где

$$
k_{s}^{2}(\xi)=\frac{\pi c}{I_{b}^{2}}\left(\frac{I_{b}}{I_{A}}\right) \int_{0}^{\infty} d r r J_{b z 0}^{2}(r, \xi)
$$

- квадрат волнового числа шланговых колебаний, $\alpha_{p h}$ - коэффициент фазового перемешивания траекторий электронов пучка.

Граничные и начальные условия для системы уравнений (5), (6) и (9) выберем следующим образом:

$$
\begin{gathered}
\left.\Psi_{f}\right|_{\xi=0}=\Psi_{b},\left.\quad \frac{\partial \Psi_{f}}{\partial \xi}\right|_{\xi=0}=0, \\
\left.Y\right|_{z=0}=0.02 R_{c h},\left.\quad \frac{\partial Y}{\partial z}\right|_{z=0}=0, \\
\left.D\right|_{\xi=0}=0,\left.\quad \frac{\partial D}{\partial \xi}\right|_{\xi=0}=0 .
\end{gathered}
$$

Система уравнений (5), (6), (9), (11) и (12) является обобщением известных уравнений (см. $[5,6,8]$ ) на ситуацию произвольной омической проводимости в плазменном канале, когда условие (1) не выполнено. 
Рассмотрим безразмерные величины

$$
\begin{gathered}
\Psi_{f 1}=\frac{\Psi_{f}}{\left(I_{b 0} / c\right)}, \quad \Psi_{b 1}=\frac{4}{S_{0}^{2}} P(\xi), \\
D_{1}=\frac{D}{R_{c h}}, \quad Y_{1}=\frac{Y}{R_{c h}}, \\
z_{1}=z k_{s}, \quad \xi_{1}=\frac{\xi}{\xi_{m}+\xi_{c}} .
\end{gathered}
$$

Тогда уравнения (5), (6), (9), (11) и (12) принимают следующий вид:

$$
\begin{gathered}
A \frac{\partial^{2} \Psi_{f 1}}{\partial \xi_{1}^{2}}+\frac{\partial \Psi_{f 1}}{\partial \xi_{1}}+\Psi_{f 1}=\Psi_{b 1}, \\
A \Psi_{f 1} \frac{\partial^{2} D_{1}}{\partial \xi^{2}}+2 A \frac{\partial \Psi_{f 1}}{\partial \xi_{1}} \frac{\partial D_{1}}{\partial \xi_{1}}=\Psi_{b 1}\left(Y_{1}-D_{1}\right), \\
\frac{\partial^{2} Y_{1}}{\partial z_{1}^{2}}=\frac{\Psi_{f 1}}{\Psi_{b 1}}\left(D_{1}-Y_{1}\right)-\alpha \alpha_{p h} \sqrt{\left|\frac{\Psi_{f 1}}{\Psi_{b 1}}\right|} \frac{\partial Y_{1}}{\partial z_{1}}, \\
\left.\Psi_{f 1}\right|_{\xi_{1}=0}=\Psi_{b 1},\left.\quad \frac{\partial \Psi_{f 1}}{\partial \xi_{1}}\right|_{\xi_{1}=0}=0, \\
\left.Y_{1}\right|_{z_{1}=0}=0.02,\left.\quad \frac{\partial Y_{1}}{\partial z_{1}}\right|_{z_{1}=0}=0, \\
\left.D_{1}\right|_{\xi_{1}=0}=0,\left.\quad \frac{\partial D_{1}}{\partial \xi_{1}}\right|_{\xi_{1}=0}=0,
\end{gathered}
$$

где $A=\xi_{m} \xi_{c} /\left(\xi_{m}+\xi_{c}\right)^{2}$.

Предположим, что все характерные параметры задачи не зависят от координаты $\xi$. Тогда неизвестные функции в рассматриваемой задаче будем искать в виде фурье-моды:

$\Psi_{f 1}(\xi)=\Psi_{f 0} e^{-i \omega_{1} \xi_{1}}, \quad D_{1}=D_{10} e^{-i \omega_{1} \xi_{1}}, \quad Y_{1}=Y_{10} e^{-i \omega_{1} \xi_{1}}$,

где $\omega_{1}=\omega\left(\xi_{m}+\xi_{c}\right)-$ безразмерная пространственная частота по координате $\xi$ и $\Psi_{f 0}, D_{10}, Y_{10}$ - безразмерные амплитуды соответствующих величин, $i$ - мнимая единица.

Будем считать, что частота $\omega_{1}$ является известной вещественной величиной.

Тогда из (15) имеем

$$
\Psi_{f 1}(\xi)=\frac{\Psi_{b 1}}{1-i \omega_{1}-A \omega_{1}^{2}} .
$$

С помощью (16) и (21) находим

$$
D_{1}=Y_{1} \frac{1-i \omega_{1}-A \omega_{1}^{2}}{1-2 i \omega_{1}-4 A \omega_{1}^{2}}
$$

После подстановки (21) и (22) в (17) получим

$$
\frac{\partial^{2} Y_{1}}{\partial z_{1}^{2}}=Y_{1}\left(\frac{a+2 i \omega_{1}}{a^{2}+4 \omega_{1}^{2}}-\frac{b+i \omega_{1}}{b^{2}+\omega_{1}^{2}}\right)-\frac{\alpha_{p h}}{\left(b^{2}+\omega_{1}^{2}\right)^{1 / 4}} \frac{\partial Y_{1}}{\partial z_{1}},
$$

где $a=1-4 A \omega_{1}^{2}, b=1-A \omega_{1}^{2}$.
Далее представим $Y_{1}=Y_{1 r}+i Y_{1 i}$, где $Y_{1 r}=\operatorname{Re}\left(Y_{1}\right)$, $Y_{1 i}=\operatorname{Im}\left(Y_{1}\right)$. Тогда, разделяя уравнение (23) на вещественную и мнимую части, находим

$$
\begin{aligned}
& \frac{\partial^{2} Y_{1 r}}{\partial z_{1}^{2}}=Y_{1 r}\left(\frac{a}{a^{2}+4 \omega_{1}^{2}}-\frac{b}{b^{2}+\omega_{1}^{2}}\right) \\
& +Y_{1 i}\left(-\frac{2 \omega_{1}}{a^{2}+4 \omega_{1}^{2}}-\frac{\omega_{1}}{b^{2}+\omega_{1}^{2}}\right)-\frac{\alpha_{p h}}{\left(b^{2}+\omega_{1}^{2}\right)^{1 / 4}} \frac{\partial Y_{1 r}}{\partial z_{1}}, \\
& \frac{\partial^{2} Y_{1 i}}{\partial z_{1}^{2}}=Y_{1 r}\left(\frac{2 \omega_{1}}{a^{2}+4 \omega_{1}^{2}}-\frac{\omega_{1}}{b^{2}+\omega_{1}^{2}}\right) \\
& -Y_{1 i}\left(-\frac{a}{a^{2}+4 \omega_{1}^{2}}-\frac{b}{b^{2}+\omega_{1}^{2}}\right)-\frac{\alpha_{p h}}{\left(b^{2}+\omega_{1}^{2}\right)^{1 / 4}} \frac{\partial Y_{1 i}}{\partial z_{1}} .
\end{aligned}
$$

Граничные условия выберем следующим образом:

$$
\begin{aligned}
& \left.Y_{1 r}\right|_{z_{1}=0}=0.02,\left.\quad Y_{1 i}\right|_{z_{1}=0}=0, \\
& \left.\frac{\partial Y_{1 r}}{\partial z_{1}}\right|_{z_{1}=0}=0,\left.\quad \frac{\partial Y_{1 i}}{\partial z_{1}}\right|_{z_{1}=0}=0 .
\end{aligned}
$$

На рисунке представлены зависимости $\left|Y_{1}\right|$ от безразмерной координаты $z_{1}$ при разных значениях частоты $\omega_{1}$. Коэффициент фазового перемешивания был взят $\alpha_{p h}=0.38$.

Из рисунка следует, что РШН имеет наибольшую амплитуду нарастания по координате $z_{1}$ в случае $\eta=\xi_{m} / \xi_{c}=1$, а наименьшую - при больших значениях параметра $\eta$. Это может быть объяснено тем, что при $\eta=1$ работают оба дестабилизирующих фактора магнитный и электростатический, тогда как в случае 1 и 2 превалируют только соответственно магнитный и электростатический факторы.

\section{Заключение}

В настоящей работе рассмотрена пространственная динамика РШН РЭП пучка в случаях, когда время зарядовой нейтрализации много больше, порядка и много меньше времени токовой компенсации. Получено, что наибольшим пространственным инкрементом нарастания указанная неустойчивость обладает в случае, когда время зарядовой нейтрализации порядка скинового времени. В этой ситуации необходимо учитывать как магнитный, так и электростатический факторы дестабилизации пучка.

\section{Список литературы}

[1] Рухадзе А.А., Богданкевич Л.С., Росинский С.Е., Рухлин В.Г. Физика сильноточных релятивистских электронных пучков. М.: Атомиздат, 1980. 167 с.

[2] Диденко А.Н., Григорьев В.П., Усов Ю.П. Мощные электронные пучки и их применение. М.: Атомиздат, 1977. $277 \mathrm{c}$. 
[3] Миллер Р. Введение в физику сильноточных пучков заряженных частиц. М.: Мир, 1984. 432 с.

[4] Колесников Е.К., Мануйлов А.С., Филиппов Б.В. Динамика пучков заряженных частиц в газоплазменных средах. СПб.: Изд-во СПбГУ, 2002. 98 с.

[5] Lee E.P. // Phys. Fluid. 1978. Vol. 21. N 8. P. 1327-1343.

[6] Надеждин Е.Р., Сорокин Г.А. // ФП. 1983. Т. 9. № 5. C. 989-991.

[7] Lee E.P. Lawrence Livermore Lab. Rep. UCID-17286. 1976. $18 \mathrm{p}$.

[8] Lampe M., Sharp W.M., Hubbard R., Lee E.P. et al. // Phys. Fluid. 1984. Vol. 27. N 12. P. 2921-2936.

[9] Кондратьев Н.А., Сметанин В.И. // ЖТФ. 2005. Т. 75. Вып. 3. С. 67-73.

[10] Мануйлов А.С. // ЖТФ. 2000. Т. 70. Вып. 1. С. 76-78.

[11] Колесников Е.К., Мануйлов А.С. // ЖТФ. 1991. Т. 61. Вып. 12. С. 43-46. 